\title{
Review of: "Isothermal amplification and fluorescent detection of SARS-CoV-2 and SARS-CoV-2 variant virus in nasopharyngeal swabs"
}

\author{
György Lengyel ${ }^{1}$ \\ 1 Semmelweis University of Medical Sciences
}

Potential competing interests: The author(s) declared that no potential competing interests exist.

Jones et al. presents a comparative study for the laboratory detection of SARS-CoV-2 that utilizes loopmediated isothermal amplification to aim faster diagnosis of COVID-19. The relevance of the topic is clear, the fourth wave of the pandemic is ongoing right now. As of the $16^{\text {th }}$ of November 2021, 253163330 confirmed cases and 5098174 COVID-related deaths were reported to the WHO [1]. Publications regarding SARS-CoV-2 diagnostic methods and comparative analyses have essential practical use, as they aid diagnostic laboratories to choose among the available methods and diagnostic kits. With increasing need for testing, and with possible reagent shortages, it is crucial to have fast, cost-efficient, and reliable methods.

\section{Experimental setup and results}

Jones et al. performed real-time RT-PCR and RT-LAMP assays on nasopharyngeal swab samples, cell culture propagated virus samples and in vitro transcribed RNA (the N-gene of the SARS-CoV-2 Wuhan-Hu-1 strain). The main strength of the publication is that the use of in vitro sample types was supplemented with clinical samples. 50 SARS-CoV-2 positive and 84 SARS-CoV-2 negative nasopharyngeal swabs were used (these results were previously determined by a clinical microbiology laboratory), with the RT-LAMP assay, $84 \%$ of the previously positive samples were positive. The limit of detection, assessed by the testing of serial dilutions of the RT-LAMP assay was $0.1 \mathrm{fg}$ of RNA (which equals 10 copies of the $\mathrm{N}$ gene sequence) of the in vitro transcribed RNA, however, when using RNA samples purified from cell-culture supernatant, the limit of detection was 100 copies (for both the RT-LAMP and the RT-qPCR method). The average Ct value of the positive nasopharyngeal swab samples was 25.7 (ranged from 16 to 34 ) using the CDC EUA RT-qPCR assay, concordance with the RT-LAMP assay was $98 \%$. However, it would be more informing, if concordance would have been calculated for different ranges of Ct values (e.g., concordance for samples with Ct values ranging 15-20, 20-25, 25-30 etc).

It is beneficial that the authors examined the specificity of the assay via testing different human coronavirus strains (OC43, 229E and NL63) from cell culture supernatant. The RT-LAMP assay did not detect neither of the 3 closely related coronavirus strains. However, there are other pathogens as well that cause similar respiratory symptoms (other coronavirus strains, influenza virus, rhinoviruses, adenoviruses 
etc.), besides those pathogens that were used in the assessment of specificity, as well as verification of negative test results would have been useful.

The applied RT-LAMP method utilizes an intercalating fluorescent dye, which is less specific than fluorescent labelled probe based methods [2]. Furthermore, since it has been stated, that mutation in one of the genes of the virus can jeopardize detection and cause false-negative results [3], thus multiplex assays (that use probes labelled with different fluorescent dyes) can overcome this problem. The authors state that the use of RT-LAMP is a fast and cost-efficient method. However, it is only cost-efficient when a house-made assay is used. Additionally, the cost of RT-LAMP based diagnostic kits with IVD certification might be higher. RT-LAMP is indeed faster, than RT-qPCR assays, however several fast RT-qPCR assays have been available, for example, the average assay time of the Oncobit RT-PCR is 55 minutes [4], thus the whole turnaround time is just slightly higher with the use of real-time RT-PCR. Extraction free methods are also faster and cheaper; however, the reaction might be affected by enzyme inhibitors and the sensitivity might be lower due to the absence of template concentration during nucleic acid extraction [5].

The results are presented in clear, detailed figures. In the discussion, the authors end the publication with detailed limitations of the study.

\section{Verdict}

The authors compared an RT-qPCR assay and an RT-LAMP techniques, this method can be easily translated into clinical microbiology in a practical approach.

\section{Advantages}

- Several types of samples (experimental and clinical samples) were compared

- Testing of in vitro transcribed RNA and propagated viruses was supplemented with the use of clinical samples

- Acceptable sensitivity and concordance between RT-qPCR and RT-LAMP

\section{Limitations}

- The RT-LAMP assay described here is singleplex, the detection of multiple targets could improve sensitivity

- The use of intercalating dye (instead of labelled probes)

- Concordance is not specified in different Ct ranges of the RT-qPCR

- For the assessment of specificity only 3 human coronavirus strains were used

\section{References}

[1] European Centre for Disease Prevention and Control, "SARS-CoV-2 variants dashboard," 2021. .

[2] N. A. Tanner, Y. Zhang, and T. C. Evans, "Simultaneous multiple target detection in real-time loop- 
mediated isothermal amplification," Biotechniques, vol. 53, no. 2, pp. 81-89, Aug. 2012, doi: $10.2144 / 0000113902$.

[3] M. Artesi et al., "A Recurrent Mutation at Position 26340 of SARS-CoV-2 Is Associated with Failure of the E Gene Quantitative Reverse Transcription-PCR Utilized in a Commercial Dual-Target Diagnostic Assay," J. Clin. Microbiol., vol. 58, no. 10, Sep. 2020, doi: 10.1128/JCM.01598-20.

[4] A. Tastanova et al., "A Comparative Study of Real-Time RT-PCR-Based SARS-CoV-2 Detection Methods and Its Application to Human-Derived and Surface Swabbed Material," J. Mol. Diagnostics, vol. 23, no. 7, pp. 796-804, Jul. 2021, doi: 10.1016/j.jmoldx.2021.04.009.

[5] M. R. Hasan et al., "Detection of SARS-CoV-2 RNA by direct RT-qPCR on nasopharyngeal specimens without extraction of viral RNA," PLoS One, vol. 15, no. 7, p. e0236564, Jul. 2020, doi:

10.1371/journal.pone.0236564. 